\title{
Preferencia manual precoz: signo de alarma de accidente cerebrovascular perinatal. Serie de casos
}

\author{
Early handedness: Warning sign of perinatal stroke. Case series
}

\author{
Dr. Sebastián Gacio ${ }^{a}$
}

\begin{abstract}
RESUMEN
Accidente cerebrovascular presuntamente perinatal es la expresión utilizada para los casos en los que se diagnostica un accidente cerebrovascular antiguo por sus secuelas y no por la sintomatología aguda. Muchos accidentes cerebrovasculares presuntamente perinatales tienen como primera manifestación una hemiparesia congénita, la cual se hacenotoria entre el cuarto y el octavo mes de vida como preferencia manual precoz. Es por esto por lo que la preferencia manual clara y persistente desarrollada antes del año de vida debe asumirse como un signo de alarma de una probable secuela motora.

En este trabajo, revisamos la historia clínica de 15 casos de accidentecerebrovascular presuntamente perinatal para evaluar la edad en la que la consulta derivó en el diagnóstico, el motivo de consulta y la edad de desarrollo de la preferencia manual. Palabras clave: accidentecerebrovascular presuntamente perinatal, hemiparesia congénita, lateralidad funcional.
\end{abstract}

\begin{abstract}
Presumed perinatal ischemic stroke is the term used for cases in which an old stroke is diagnosed by the consequences of it and not by the acute symptoms. Many presumed perinatal ischemic strokes have congenital hemiparesis as the first manifestation, which is usually noticed between the fourth and eighth month of life as early hand preference. That is why the clear and persistent handedness developed before one year of age must be assumed as a warning sign of probable motor sequelae. In this paper we review the medical records of 15 cases of presumed perinatal ischemic stroke to assess the age at which the consultation led to the diagnosis, reason for consultation and age at development of handedness.

Key words: presumed perinatal ischemic stroke, congenital hemiparesis, handedness.
\end{abstract}

http:/ / dx.doi.org/10.5546/aap.2016.e374

a. Instituto de Neurología Cognitiva (INECO) e Instituto de Neurociencias, Universidad Favaloro.

Ciudad Autónoma de Buenos Aires. Argentina.

Servicio de Pediatría, Hospital Juan A. Fernández.

Ciudad Autónoma de Buenos Aires. Argentina.

Neurología Infantil. Sanatorio Fueguino. Río Grande,

Tierra del Fuego. Argentina.

Correspondencia:

Dr. Sebastián Gacio, sgacio@ineco.org.ar.

Financiamiento: Ninguno.

Conflicto de intereses: Ninguno que declarar.

Recibido: 2-3-2016

Aceptado: 13-4-2016

\section{INTRODUCCIÓN}

La etapa perinatal es uno de los momentos de la vida de mayor riesgo de sufrir un accidente cerebrovascular (ACV), con una incidencia de 1 caso cada $1600-5000$ nacimientos. ${ }^{1-3} \mathrm{La}$ principal manifestación clínica de esta entidad son las convulsiones; sin embargo, muchos recién nacidos que sufrieron un $\mathrm{ACV}$ prenatal o neonatal no manifestarán signos sugerentes y, en consecuencia, el ACV se diagnosticará tiempo después, debido a sus secuelas y no a la sintomatología aguda. Para esta situación en la cual el diagnóstico de ACV perinatal se difiere meses o años y se realiza fuera del período neonatal, se ha adoptado la expresión accidente cerebrovascular isquémico presuntamente perinatal (Presumed Perinatal Ischemic Stroke; PPIS, por sus siglas en inglés). ${ }^{4}$

La edad de presentación y/o diagnóstico del PPIS va a depender del territorio cerebral afectado $y$, en consecuencia, de la secuela producida. ${ }^{5}$ Debido a que las dos lesiones más frecuentes son los ACV de arteria cerebral media y los infartos venosos periventriculares (Figura 1), y ambas comprometen áreas motoras (ya sea corteza motora o tractos motores en la sustancia blanca), la mayoría de los niños con PPIS se diagnostican entre los 4 y los 8 meses de vida cuando comienza a hacerse manifiesta la hemiparesia congénita secuela del ACV. Sin embargo, algunos niños con lesiones que comprometen áreas motoras son diagnosticados luego del año de vida al iniciar la marcha.

Un signo temprano de hemiparesia congénita es el desarrollo precoz de la preferencia manual. La mayoría de los niños desarrollan su preferencia manual alrededor de los 2 años de vida. Si bien, a partir del año, puede observarse una preferencia incipiente hacia el uso de una de sus manos, la preferencia manual clara y persistente dentro del año de vida debe ser interpretada como un signo de alarma de una probable hemiparesia del miembro contralateral. ${ }^{6}$ Como la principal causa de hemiparesia congénita son los ACV perinatales, ante un niño con preferencia manual clara dentro del año de vida, debe sospecharse, en 
primer lugar, la posibilidad de que haya sufrido un ACV en la etapa perinatal.

Existen, sin embargo, niños con PPIS asociado a hemiparesia que son diagnosticados después del año al notar anomalías en la marcha.

Para este trabajo, se formuló la hipótesis de que muchos de estos niños con diagnóstico de PPIS por alteraciones de la marcha habían desarrollado una preferencia manual clara antes de los 12 meses de vida, pero que no había sido considerada como patológica en ese momento.

El objetivo del estudio es presentar una serie de 15 niños con diagnóstico de PPIS en relación con el momento de aparición de su preferencia manual claramente definida.

\section{MÉTODO}

Para confirmar la hipótesis, se revisaron las historias clínicas de 15 niños con diagnóstico de PPIS, todos ellos evaluados por el autor en el Instituto de Neurociencias de la Fundación Favaloro, el Sanatorio Fueguino y el Hospital Fernández entre enero de 2011 y diciembre de 2015. Se revisó la edad al momento del diagnóstico y el motivo de consulta que llevó a la obtención de la neuroimagen, así como la edad en la que los padres notaron una preferencia manual claramente definida.

El trastorno motor se definió como preferencia manual precoz (PMP) o anomalía de la marcha (AM). Para los fines prácticos del trabajo, se asumió como normal cuando la preferencia manual fue notada después del primer año de vida.

\section{RESULTADOS}

Los 15 niños con diagnóstico de PPIS fueron incluidos en el estudio (Tabla 1).

La edad de presentación varió de 2 meses a 11 años, pero, 11 de los 15 casos se presentaron dentro de los 18 meses de vida.

Diez de los 15 casos consultaron por anomalías motoras. El resto de los pacientes tuvieron como primera manifestación convulsiones (3/15) o retraso global del desarrollo (RGD) (2/15). Los 3 niños con convulsiones tuvieron su primera crisis a los 2 meses, 18 meses y 11 años. Los niños con RGD fueron diagnosticados a los 10 meses y a los 2 años y medio.

De los 10 niños con compromiso motor inicial, 8 tuvieron ACV arteriales, todos por compromiso de la arteria cerebral media (ACM) (3 de arterias lentículo-estriadas) y 2 pacientes tuvieron infartos venosos. Cinco niños fueron traídos a la consulta entre los 3 y los 9 meses de vida por sospecha de hemiparesia congénita, debido al uso preferencial de uno de sus brazos (PMP), y los restantes 5 casos consultaron entre los 12 meses y los 3 años y medio de edad por anomalías notadas al iniciar la marcha (AM).

$\mathrm{Al}$ interrogar a los padres de los niños con presentación motora que consultaron después del año de vida por anomalías en la marcha, en todos los casos (5/5), se refirió la presencia de preferencia manual claramente desarrollada antes de los 12 meses de vida (véase la Tabla 1), pero no había llamado la atención para hacer la consulta o no había sido interpretada como un signo de alarma. El retraso en el diagnóstico

FIGURA 1. En la figura, se muestran ejemplos de resonancia magnética de los distintos tipos de ACV isquémicos presuntamente perinatales. A (caso 6): ACV isquémico arterial por compromiso de la ACM derecha. B (caso 7): ACV isquémico arterial por compromiso de las arterias lentículo-estriadas derechas, ramas de la ACM (círculo). C (caso 9): ACV isquémico venoso izquierdo (infarto venoso periventricular). Se observa la lesión de la sustancia blanca periventricular con dilatación del ventrículo lateral adyacente (círculo).

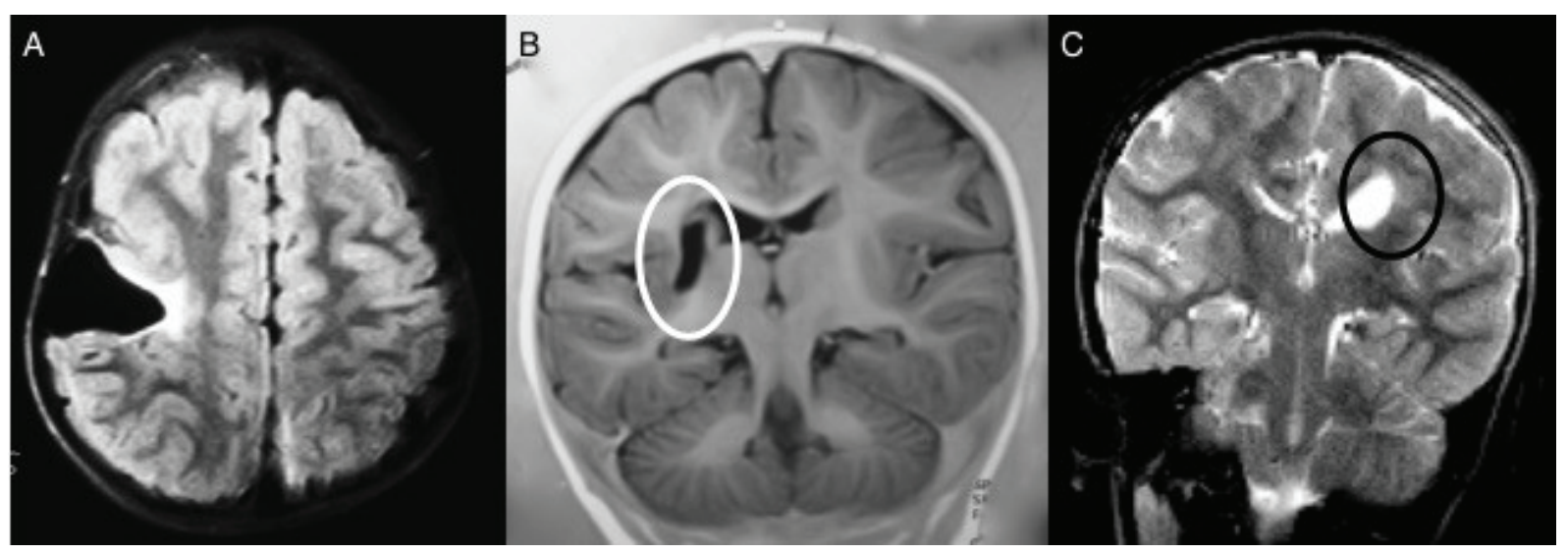


desde el momento en que los padres notaron la preferencia manual y el diagnóstico definitivo de PPIS de estos 5 casos varió entre 4 meses (caso 7) y 2 años y 11 meses (caso 9). Todos los niños que consultaron por convulsiones o RGD tuvieron preferencia manual desarrollada después del año.

\section{DISCUSIÓN}

PPIS es la expresión utilizada para definir el hallazgo de un ACV antiguo al estudiar pacientes por los síntomas que son su secuela y no la sintomatología aguda. ${ }^{7}$

Los dos principales motivos de consulta de niños con PPIS son la hemiparesia congénita y las convulsiones sintomáticas remotas; la primera es más frecuente. ${ }^{8}$ Las hemiparesias congénitas suelen comenzar a manifestarse entre el cuarto y el octavo mes de vida cuando el niño comienza a utilizar las manos para tomar objetos. En ese momento, se observa una clara preferencia por utilizar uno de sus brazos, lo cual lleva a los padres a notar que es claramente derecho o izquierdo. La preferencia manual desarrollada antes del año es un signo de alarma de una probable hemiparesia congénita $y$, en consecuencia, de un ACV perinatal o, menos frecuentemente, una malformación del desarrollo cortical.
En ocasiones, la hemiparesia es notada por primera vez con el inicio de la marcha luego del año de vida. Es frecuente que estos niños tengan también paresia braquial y que esta no haya sido advertida previamente. ${ }^{6}$

Todos nuestros casos de PPIS con hemiparesia que fueron diagnosticados luego del año debido a las alteraciones en la marcha tenían preferencia manual ya notada por los padres antes del año de vida, pero no asumida como un signo de alarma.

Si bien el retraso en el diagnóstico del ACV agudo ya se produjo en el momento de hacer el diagnóstico de PPIS, la demora en el diagnóstico de esta entidad retrasa también el inicio de las terapias destinadas a mejorar las secuelas del niño, así como la evaluación etiológica destinada a disminuir el riesgo de recurrencia.

En uno de los casos presentados, se demoró más de 2 años entre el momento de la observación de la preferencia manual y el diagnóstico final.

Aunque el tamaño de la muestra no permite obtener conclusiones estadísticamente significativas, de lo observado en esta serie, podemos resaltar la importancia de evaluar, en la consulta clínica, la PMP y asumirla como un signo de alarma de hemiparesia congénita $y$, en consecuencia, de un probable ACV perinatal subdiagnosticado.

TABla 1: Descripción de los pacientes con accidente cerebrovascular presuntamente perinatal

\begin{tabular}{lcccccc}
\hline Paciente & Tipo de ACV & Vaso & Localización & Edad & Síntoma & Edad de preferencia manual \\
\hline 1 & A & ACM & B & $3 \mathrm{~m}$. & PMP & $3 \mathrm{~m}$. \\
2 & A & ACM & D & $8 \mathrm{~m}$. & PMP & $8 \mathrm{~m}$. \\
3 & A & ACM & D & $4 \mathrm{~m}$. & PMP & $4 \mathrm{~m}$. \\
4 & A & LS & I & $9 \mathrm{~m}$. & PMP & $9 \mathrm{~m}$. \\
5 & A & ACM & D & $8 \mathrm{~m}$. & PMP & $8 \mathrm{~m}$. \\
6 & A & ACM & D & $21 \mathrm{~m}$. & AM & $6 \mathrm{~m}$. \\
7 & A & LS & D & $12 \mathrm{~m}$. & AM & $6 \mathrm{~m}$. \\
8 & A & LS & I & $14 \mathrm{~m}$. & AM & $11 \mathrm{~m}$. \\
9 & V & - & I & 3 a., $6 \mathrm{~m}$. & AM & Normal \\
10 & V & - & D & $18 \mathrm{~m}$. & AM & Normal \\
11 & A & ACP & I & $18 \mathrm{~m}$. & CNV & Normal \\
12 & A & ACM & I & $11 \mathrm{a}$. & CNV & Normal \\
13 & V & - & B & $2 \mathrm{~m}$. & CNV & Normal \\
14 & A & LS & D & $10 \mathrm{~m}$. & RGD & RGD \\
15 & V & - & I & 2 a., $6 \mathrm{~m}$. & R & \\
\hline
\end{tabular}

Edad: edad en la que se realizó la consulta que derivó en el diagnóstico. ACV: accidente cerebrovascular. A: arterial. V: venoso. ACP: arteria cerebral posterior. ACM: arteria cerebral media. LS: arterias lentículo-estriadas. B: bilateral. D: derecho. I: izquierdo. PMP: preferencia manual precoz. AM: anomalía de la marcha. CNV: convulsiones. RGD: retraso global del desarrollo. a.: años. m.: meses. 
No está bien definido el plan de estudio de niños con ACV perinatales, cuya recurrencia, además, es muy baja, pero se sugiere descartar cardiopatías congénitas, ya que son una de la principales causas. Es más controvertido el estudio de anomalías protrombóticas, aunque vale remarcar que, en la serie de casos de Kocaman y Yilmaz, el 69\% de los niños tenían, al menos, un factor de riesgo protrombótico; el más frecuente fue la mutación del gen metiltetrahidrofolato redactasa (MTHFR). ${ }^{8}$

Debido a que las secuelas de lesiones perinatales pueden aparecer con el tiempo, el diagnóstico de PPIS obliga a iniciar un seguimiento estricto del neurodesarrollo para diagnosticar sus secuelas de manera temprana y tratarlas oportunamente conociendo las ventajas de la intervención precoz en niños gracias a la neuroplasticidad propia del desarrollo cerebral infantil. ${ }^{9,10}$

\section{CONCLUSIONES}

El ACV perinatal es la principal causa de hemiparesia congénita. En los niños con diagnóstico de PPIS con compromiso motor, la preferencia manual precoz desarrollada antes del año de vida es el primer signo de alarma para el médico pediatra, $\mathrm{y}$, por ende, su reconocimiento brinda la posibilidad de un diagnóstico temprano $\mathrm{y}$, en consecuencia, un tratamiento oportuno.

\section{REFERENCIAS}

1. Lynch JK. Epidemiology and classification of perinatal stroke. Semin Fetal Neonatal Med 2009;14(5):245-9.

2. Schulzke $S$, Weber $P$, Luetschg J, Fahnenstich H. Incidence and diagnosis of unilateral arterial cerebral infarction in newborn infants. J Perinat Med 2005;33(2):170-5.

3. Nelson KB. Perinatal ischemic stroke. Stroke 2007;38(2 Suppl):742-5.

4. Raju TN, Nelson KB, Ferriero D, Lynch JK. Ischemic perinatal stroke: summary of a workshop sponsored by the National Institute of Child Health and Human Development and the National Institute of Neurological Disorders and Stroke. Pediatrics 2007;120(3):609-16.

5. Kirton A, DeVeber G, Pontigon AM, Macgregor D, et al. Presumed perinatal ischemic stroke: vascular classification predicts outcomes. Ann Neurol 2008;63(4):436-43.

6. Kirton A, Shroff M, Pontigon AM, DeVeber G. Risk factors and presentations of periventricular venous infarction vs arterial presumed perinatal ischemic stroke. Arch Neurol 2010;67(7):842-8.

7. Gacio S, Muñoz Giacomelli F, Klein F. Accidente cerebrovascular isquémico presuntamente perinatal. Revisión. Arch Argent Pediatr 2015;113(5):449-55.

8. Kocaman C, Yilmaz Y. Etiological analysis of presumed perinatal stroke. Brain Dev 2012;34(2):133-9.

9. Kirton A. Modeling developmental plasticity after perinatal stroke: defining central therapeutic targets in cerebral palsy. Pediatr Neurol 2013;48(2):81-94.

10. Basu AP. Early intervention after perinatal stroke: opportunities and challenges. Dev Med Child Neurol 2014;56(6):516-21. 